Microgravity Sciences and Processes Symposium (A2.)

Fluid and Materials Sciences (2.)

Author: Dr. Shumpei Ozawa

University of Tokyo, Hino, Japan, shumpei.ozawa@tmit.ac.jp

Dr. Satoshi Hirosawa

Research and Development Center, NEOMAX Co., Ltd, Osaka, Japan,

HIROSAWA.S@neomax.co.jp

Dr. Kazuhiko Kuribayashi

Japan Aerospace Exploration Agency (JAXA) / ISAS, Sagamihara, Japan, kuribayashi@isas.jaxa.jp

Dr. Sven Reutzel

Institute of Space Simulation, German Aerospace Center (DLR), Cologne, Germany, sven.reutzel@dlr.de

Prof. Dieter Herlach

Institute of Space Simulation, German Aerospace Center (DLR), Cologne, Germany, dieter.herlach@dlr.de

Prof. Taketoshi Hibiya

University of Tokyo, Tokyo, Japan, hibiya@cc.tmit.ac.jp

\title{
TRANSFORMATION OF ND-FE-B METASTABLE PHASE SOLIDIFIED FROM UNDERCOOLED MELT
}

\begin{abstract}
To improve magnetic properties of the Nd-Fe-B magnets, it is crucial to eliminate soft magnetic inclusions such as soft magnetic $\alpha$-Fe phase. Recently, several efforts have been devoted to producing the Nd-Fe-B alloys free from the $\alpha$-Fe phase by undercooling solidification. In these works, it was expected that the direct crystallization of the Nd2Fe14B phase would suppress the formation of the $\alpha$-Fe phase. Gao and co-workers, however, suggested that the metastable $\mathrm{Nd} 2 \mathrm{Fe} 17 \mathrm{Bx}$ phase is formed as the primary phase [1]. In addition, they showed that, even in the Nd14Fe79B7 alloy, in which the a-Fe phase is unstable, the $\mathrm{Nd} 2 \mathrm{Fe} 17 \mathrm{Bx}$ phase decomposes into the $\mathrm{Nd} 2 \mathrm{Fe} 14 \mathrm{~B}$ and $\alpha$-Fe phases at the post-recalescence cooling stage. Our previous works showed that the post-processing heat-treatment transforms the metastable phase into the Nd2Fe14B phase without forming the $\alpha$-Fe phase [2]. These results imply that the kinetics of the transformation in the metastable phase at the post-recalescence cooling stage is different from that at the post-solidification heat-treatment. In the present study, the undercooling solidification of $\mathrm{NdxFe}(100-1.5 \mathrm{x}) \mathrm{B} 0.5 \mathrm{x}$ alloys was carried out in a drop tube. The objective of this investigation is to clarify the mechanism of the phase transformation in the rapidly solidified Nd-Fe-B alloys from the undercooled melt. Segments of ingot were induction melted in a helium atmosphere, and the molten alloy was then ejected into the $26 \mathrm{~m}$ drop tube to obtain the metastable phase. The changes of the microstructure and the phase constituent during the heat-treatment of the as-dropped sample were carefully investigated as a function of sample diameters. A large amount of the $\mathrm{Nd} 2 \mathrm{Fe} 17 \mathrm{Bx}$ metastable phase was contained in the as-dropped samples. The heat-treatment of the as-dropped
\end{abstract}


samples induces a dual-stage phase transformation. The 1st stage of the phase transformation is the diffusive phase transformation from $\mathrm{Nd} 2 \mathrm{Fe} 17 \mathrm{Bx}$ and Nd-rich phases into the $\mathrm{Nd} 2 \mathrm{Fe} 14 \mathrm{~B}$ phase at $950 \mathrm{~K}$, and the 2nd stage of the transformation is the decomposition of the metastable phase into the $\mathrm{Nd} 2 \mathrm{Fe} 14 \mathrm{~B}$ and $\alpha$-Fe phases at $1100 \mathrm{~K}$. Although both stages of the phase transformation is controlled by the diffusion of $\mathrm{Nd}$ atoms, transformation temperature for the 1st stage is decreased due to the large existence of the Nd-rich phase adjacent to the metastable phase.

[1] J. Gao, T. Volkmann and D. M. Herlach, Acta Mater. 50, 3003 (2002) [2] S. Ozawa, M. Li, S. Sugiyama, I. Jimbo, S. Hirosawa and K. Kuribayashi, Mater. Sci. Eng. A. 382, 295 (2004) 